\title{
The speed of change: towards a discontinuity theory of immunity?
}

\author{
Thomas Pradeu ${ }^{1 *}$, Sébastien Jaeger ${ }^{2,3,4}$ \& Eric Vivier ${ }^{2,3,4,5^{*}}$
}

Draft July 26, 2013

\section{Published version in Nature Reviews Immunology available here: http://www.nature.com/nri/journal/v13/n10/abs/nri3521.html}

${ }^{1}$ Université Paris-Sorbonne

${ }^{2}$ Centre d'Immunologie de Marseille-Luminy (CIML), Aix Marseille Université, UM2, Campus de Luminy, 13288 Marseille, France

${ }^{3}$ INSERM, U 1104, 13288 Marseille, France

${ }^{4}$ CNRS, UMR7280, 13288 Marseille, France

5 Assistance Publique des Hôpitaux de Marseille, Hôpital de la Conception, Marseille, France

*To whom correspondence should be addressed. E-mails: thomas.pradeu@paris-sorbonne.fr (T. P.); vivier@ciml.univ-mrs.fr (E.V.).

\section{Preface}

Immunology, though deeply experimental in everyday practice, is also a theoretical discipline. Recent advances in the understanding of innate immunity, how it is triggered, and how it shares features previously uniquely ascribed to the adaptive immune system, can contribute to the refinement of immunology's theoretical framework. In particular, natural killer (NK) cells 
and macrophages are activated by transient modifications, but adapt to long-lasting modifications that occur in the surrounding tissue environment. This process allows the maintenance of self-tolerance while permitting efficient immune responses. Extending this idea to other components of the immune system, we propose here some general principles that lay the ground for a unifying account of immunity, the discontinuity theory. According to this theoretical framework, effector immune responses (i.e., activated responses that lead to the potential elimination of the target antigen) are triggered by an antigenic discontinuity, that is, by the sudden modification of molecular motifs with which immune cells interact.

\section{Introduction}

For more than a century, immunologists have elaborated theories to provide descriptions, explanations and predictions about how an immune response is triggered. So much interest in theories in a field of molecular biology is somewhat uncommon ${ }^{1}$, but it has undoubtedly been very fruitful. Yet, confronted with the difficulties encountered by some of these theories ${ }^{2}$, several immunologists might be tempted to abandon the ambition to build what they see as grand theories, preferring instead to focus on the elucidation of the molecular mechanisms of immune responses. Nevertheless, theories are essential to interpret observations and develop new research hypotheses (BOX 1). We are therefore convinced that the elaboration of theories should remain associated with advances in immunology. In this Essay, we propose a new theoretical framework that aims to explain how immune cells integrate spatial, temporal, quantitative and qualitative signals to mount a response. We start with the case of natural killer (NK) cells, and then extend our framework to other immune cells. 


\section{The dynamics of natural killer cell reactivity}

NK cells are innate lymphoid cells that produce cytokines and can be cytolytic ${ }^{3,4}$. They distinguish their cellular targets from healthy cells via a panel of activating and inhibitory cell surface receptors, as well as adhesion molecules. Most NK cell activating receptors recognize cell surface ligands that are induced on modified cells, for example following tumor transformation, microbial infection, or physical and chemical assaults ${ }^{5}$. This recognition of dysregulated self allows NK cells to be selectively activated by host cells that have undergone molecular modifications, and are in some way stressed. Prototypical examples of NK cell surface receptors that function according to this mode of recognition include NKG2D and NKp30. In contrast, NK cell inhibitory receptors recognize cell surface molecules that are readily expressed at steady-state on most cells, such as killer cell immunoglobulin-like receptors (KIRs) and Ly49 molecules that recognize MHC class I molecules in humans and mice, respectively. This mode of recognition allows NK cells to be selectively activated by cells that have received stress signals leading to the downregulation of constitutively expressed molecules, such as the loss of MHC class I expression upon cytomegalovirus infection, and is thus referred to as 'missing self' recognition ${ }^{6}$. By integrating activating and inhibitory signals upon encountering a given interacting cell, NK cells thus spare normal cells and contribute to the elimination of cells expressing strongly modified motifs (FIG. 1A). Importantly, the responsiveness of NK cells decreases in the case of a chronic engagement of various activating receptors, such as NKG2D, human activating KIRs (e.g. KIR2DS1) or mouse activating Ly49 molecules (e.g. Ly49H) (FIG. 1B). Similarly, whereas NK cells grown in a MHC class I-sufficient environment can eliminate MHC class I-negative cells, NK cells grown in or transferred to a MHC class I-deficient environment are no longer able to do so (FIG. 1C) $)^{7,8,9,10}$. Thus, NK cells detect sudden modifications of their environment, but when modified motifs are long lasting, NK cells adapt to them by ceasing to be responsive. 
These observations lay the ground for a new theoretical framework, the 'discontinuity theory', proposing that immune cells trigger an effector response when there is a discontinuity in the molecular motifs with which their receptors interact, while they tend to become tolerant to continuously expressed motifs.

\section{Extending the discontinuity theory to other immune cells}

Several families of innate immune cells also respond to a sudden modification of their ligands. Macrophages, in particular, respond not only to microorganisms but also to modified host cells $s^{11,12,13}$. The molecular targets in such cases include pre-expressed host molecules that show biochemical modifications such as oxidation in the sugar chains of glycoproteins and glycolipids, molecules that show neo-expression as a result of cellular stress such as thrombospondin 1, or intracellular molecules that relocalize to the cell membrane after injury such as phosphatidylserine, DNA, endoplasmic reticulum-resident proteins or F-actin ${ }^{14,15}$. Crucially, macrophages also adapt to long-lasting motifs in their environment. This adaptation is illustrated by the well-known phenomenon of endotoxin tolerance, a process by which by which cells exposed to low concentrations of endotoxin become hyporesponsive to further challenges with endotoxin, thereby avoiding the potentially harmful effects caused by the continual presence of pro-inflammatory $\operatorname{stimuli}^{16}$. Along the same line, phagocytosis of potential target cells is blocked when macrophages detect CD47 (a transmembrane protein that is ubiquitously expressed by host cells) via the inhibitory receptor signal-regulatory protein $1 \alpha(\mathrm{SIRP} 1 \alpha)$. As a consequence, when a macrophage encounters a CD47-deficient erythrocyte, it phagocytoses it, as the inhibitory macrophage receptor SIRP1 $\alpha$ is no longer engaged by CD47. However, macrophages from CD47-deficient mice do not phagocytose CD47-deficient erythrocytes ${ }^{17}$. Hence, the discontinuity theory applies very well to 
macrophages, which sense changes in their environment but adapt to these changes when they become persistent.

Could the same concept be extended to other immune cells, such as T and B cells? The activation of these lymphocytes is admittedly much more complex, in particular because they undergo a thorough selection process in primary lymphoid organs where, in theory, potentially self-reactive lymphocytes are removed. Yet several data hint at a possible discontinuity-based mechanism of immune activation in $\mathrm{T}$ and $\mathrm{B}$ cells. In particular, it is well established that conventional $\mathrm{T}$ and $\mathrm{B}$ cells are anergized when an antigen is chronically or constantly present ${ }^{18,19}$. For instance, several chronic infections cause functional exhaustion or deletion of antigen-specific conventional $\alpha \beta \mathrm{T}$ cells ${ }^{20,21}$. Similarly, the chronic exposure of non-conventional mouse $\gamma \delta \mathrm{T}$ cells $(\mathrm{V} \gamma 5 \mathrm{~V} \delta 1)$ to Skint1, a prototypic member of a novel family related to butyrophilin-like molecules, led to their hyporesponsiveness ${ }^{22}$. All these observations may contribute to a renewal of the 'tunable activation threshold' model, according to which lymphocytes sense relative changes, rather than absolute strength, and respond only if a sufficiently rapid increase in their level of stimulation occurs ${ }^{23,24}$. Undoubtedly, more work will be needed to determine whether the discontinuity theory applies to $\mathrm{T}$ and $\mathrm{B}$ cells. For the moment, suffice it to say that this theory aims primarily at explaining innate immune responses, which is key to understanding immune responses in general ${ }^{25}$.

Lastly, an area in which the framework presented here seems particularly relevant is that of tumour immunology. It is now well established that tumours give rise to effector immune responses ${ }^{26,27,28,29,30}$. Though tumour cells are, genetically speaking, self cells, they are transformed cells that possess several features that distinguish them from healthy host cells, particularly the expression of abnormal motifs at their cell surface ${ }^{28,31}$. Effector immune responses to tumours can be explained by the apparition of these strongly modified patterns. 
Conversely, several experiments showed that a persistent tumour antigen diminishes the efficacy of the antitumour response $\mathrm{e}^{32,33}$.

\section{The core of the discontinuity theory}

\section{Antigenic discontinuity}

We believe that a very simple theoretical framework can unify under a single explanatory principle the diverse immunological data presented above. We call this framework the 'discontinuity theory'. In a nutshell, it states that effector immune responses are triggered by an antigenic discontinuity, that is, by the appearance of molecular motifs that are qualitatively or quantitatively different from those with which the immune system has regularly interacted so far. Note that an antigen is defined here as any entity with which immune receptors, innate or adaptive, interact. The criterion of immunogenicity that we adopt is antigenic discontinuity itself, and not the origin of the antigen: indeed, a discontinuity can appear at the level of self motifs (for example, a tumour), as well as non-self motifs (for example, a microbe) ${ }^{34}$. From this point of view, the framework presented here echoes the concepts of immune surveillance ${ }^{35,36,37}$, and altered self $^{38,39}$, incorporating them in a more comprehensive perspective. More generally, the idea that antigenic discontinuity is immunogenic can be related to the common and well supported principle according to which biological entities react to variations in stimuli, as it has been demonstrated for the sight for instance ${ }^{40,41,42}$.

\section{A response based on the variation of the quantities of antigens with regard to time}

The central statement of the discontinuity theory is that an effector immune response occurs as a function of the variation of quantities of antigen (dQ) with regard to time (dt), that is the value $\mathrm{dQ} / \mathrm{dt}$ (BOX2 details how the variation can be modeled with respect to time). Indeed, two key factors in the triggering of an immune response are the speed at which antigens 
appear, and the duration of antigen exposure. In addition, two aspects must be taken into account. First, the spatial aspect: the localization of the immune interaction is pivotal, as immune responses are regulated by the local environment, which is often characterized by the prevalence of different cell types (for example, the gut or the skin are very different in terms of their cellular composition). Therefore, what is important is the local antigenic discontinuity, because a given antigen can be immunogenic or not according to its localization. Second, the structural aspect: the degree of molecular difference between usual antigens with which immune receptors interact and the antigen under consideration is important.

Now, why should the immune response be understood as a function of the variation of quantities of antigen with regard to time? In many cases, antigens appearing very progressively do not represent a strong modification and hence are not eliminated ${ }^{43,44}$. Motifs changing at a very high rate can make protective immune responses impossible, as happens with many parasites and viruses including $\mathrm{HIV}^{45,46,47}$. Indeed, in these conditions, the time required for the generation of protective immune response is greater than the time needed for the pathogens to mutate. As a consequence, these immune responses are constantly inadequate to the pathogen. The duration of the antigen exposure is also crucial, as cells exposed to chronic stimulations become desensitized by various mechanisms. Desensitization is by no means specific to immunity. In all living objects, the detection of changes in the environment of a cell (for example, the recognition of a stimulus by a cell surface receptor) is associated with the initiation of regulatory pathways that are either intrinsic or extrinsic to the stimulated cell. Desensitization of signaling pathways is a common cell intrinsic regulatory mechanism, and is an emerging property resulting from feedback and/or feedforward loops ${ }^{48}$. The mechanisms used to achieve desensitization include cell surface receptor internalization (for example, $\mathrm{T}$ cells and $\mathrm{B}$ cells internalize their antigen receptors following antigen 
recognition) and the degradation of key downstream signaling elements. Other cell intrinsic regulatory circuits include the activation of enzymatic pathways that counteract the signaling cascade initiated by the initial stimulus. For example, receptors bearing immunoreceptor tyrosine-based inhibition motifs (ITIMs) recruit and activate phosphatases that antagonize the activating kinases triggered by the receptors bearing immunoreceptor tyrosine-based activation motifs (ITAMs) ${ }^{49}$. There are also several cell extrinsic regulatory pathways that are at work, such as the regulatory $\mathrm{T}$ cells (Tregs) that negatively control $\mathrm{T}$ cell activation $^{50,51,52}$. More ambitious developments of the theory such as considering more refined modes of discontinuity detection mechanisms or feedback/forward could be explored, but each of these refinements will also need efforts on the experimental side to generate suitable (dynamical) results, identify crucial questions and refine progressively our knowledge. In particular, whether the extrinsic control exerted by Tregs on effector cells is also governed by mechanisms that are described by the discontinuity theory has to be addressed experimentally to offer an adequate picture of this issue. However, this illustrates what is perhaps the most valuable benefit in having a theory: focusing investigations on most pertinent and informative hypotheses.

The main claims made by the discontinuity theory can be summarized as follows. The sudden appearance of a structurally different motif triggers a strong effector immune response; it ends when the antigen is eliminated, and may lead to the generation of memory cells (FIG. 2A). An initially unusual but persistent motif will first trigger an effector immune response, but then the chronic presence of the motif will lead to the extinction of the response (FIG. 2B). The slow appearance of a structurally different motif leads to a very limited reaction, and eventually to the tolerance of this motif (FIG. 2C). 


\section{Why is the discontinuity theory useful?}

\section{A unifying framework}

A new theoretical framework is accepted only if it offers both a new way of seeing things, and original experimental predictions (BOX 1). A major driving force in the dynamics of scientific theories is their growing unifying power: scientists often seek frameworks that are more comprehensive than their predecessors ${ }^{53}$. It is therefore important to emphasize that the discontinuity theory gathers under a simple explanation a range of phenomena that have traditionally received distinct and heterogeneous explanations. For example, effector immune responses to tumour cells find ad hoc explanations within the self-nonself theory, through the idea of the 'altered self' ${ }^{39}$, and the 'danger theory' ${ }^{54}$, whereas the discontinuity theory states that tumours trigger effector immune responses because they bear unusual motifs. When discontinuities in self components arise within host tissues (for example, during tumour development or cellular stress), an effector immune response does occur, and when non-self components are presented to the immune system in a progressive, continuous way (for example, commensal bacteria $\left.{ }^{55,56}\right)$, it often induces regulatory mechanisms ${ }^{57}$, and it is not destroyed by the immune system. Overall, the discontinuity framework should not be perceived as opposing previous theories (in particular the 'self versus non-self' and 'danger' theories) but rather as complementing and unifying them.

\section{Predictions and challenges}

The most important challenge is to determine how the discontinuity theory could help in the current field of immunology from a practical point of view. A key prediction made by the discontinuity theory is that chronicity diminishes the intensity of the immune response. Indeed, according to the above analysis of the dynamics of the immune response, components that persist for a significant period of time in the organism will tend to become increasingly 
tolerated by the immune system. It is however important to mention that there are other mechanisms at work in tolerance, and that acute antigenic challenges might also be tolerogenic ${ }^{58}$. Nevertheless, this principle leads to several original and testable predictions.

First, autoimmunity, which is a chronic disorder resulting from the long-lasting activation of the immune system, could appear as a major counterexample. Yet our framework predicts two possibilities for the maintenance of chronic autoimmune disorders: autoantigens are changing over the course of the illness, resembling the antigenic drift observed in influenza virus infection ${ }^{59}$, and/or the presentation of autoantigens (i.e., their contextual interaction with immune effectors) is not in fact continuous, but rather oscillating (FIG. 2D). Obviously, this hypothesis remains to be experimentally tested.

Second, some massive modifications in the host, for example, such as what occurs during gestation or puberty in mammals, or during metamorphosis in insects or amphibians, do not appear to be immunogenic, in apparent conflict with the discontinuity theory. Our prediction is that the absence of a destructive immune response in these circumstances can be explained by the fact that the immune system interacts with only small quantities of these modified antigens, and in a progressive manner ${ }^{60}$, involving regulatory rather than activating processes, as documented in the case of metamorphosis ${ }^{61}$.

Finally, a recent study challenges the rational for the use of incomplete Freund's adjuvant (IFA) emulsions for cancer vaccination. The study found that IFA emulsions promote antigen persistence at the vaccination site, thereby inducing $\mathrm{T}$ cells with an 'exhausted' phenotype that eventually $\operatorname{die}^{62}$ at these sites rather than promoting their mobilization to the tumor sites where they are needed. In addition, repeated oral administration of antigen can induce tolerance and hence desensitize children with egg allergy ${ }^{63}$. These studies are in remarkable agreement with the discontinuity theory. 


\section{Conclusions and perspective}

In an attempt to assess the discontinuity theory, we sought to develop a model that translates the main concept of this theoretical framework into a mathematical formalism. As described above, the fundamental idea here is that an effector immune response is triggered by a discontinuity in a steady state considered as the self-referential environment. We therefore generated a dynamic model with an input variable $\mathrm{E}(\mathrm{t})$ encompassing all external signals sensed by an immune cell and an output variable $\mathrm{R}(\mathrm{t})$ quantifying the immune response intensity triggered by the cell (BOX 2). Despite its simplicity, this model perfectly fits the principles of the discontinuity theory, while presenting them in a more formal, and therefore testable, way. In the current model, multiple signals are modeled by a single input variable; while we have also developed a more complex version with a multidimensional variable describing different stimuli, we cannot at present assess its accuracy due to a lack of data and will therefore not discuss it here.

We have thus tried here to lay the foundations for a new theory to explain how the immune system integrates spatial, qualitative, quantitative and temporal signals to generate an appropriate immune response. We suggest that the immune system has been shaped by natural selection for its capacity to perceive sudden antigenic modifications. This entails that the immune system usually deals efficiently with acute, but not with long lasting disruptions such as chronic infections and cancers. This view of the immune system gives rise to several predictions, as illustrated above through a few examples. As it stands, the discontinuity theory can apply to several biological scales, the cell and the populations of cells as well as the entire organism. Obviously, experimental testing will infirm or confirm the extent of its perimeter. More generally, there is little doubt that this theoretical framework will need to be amended and improved; the best contribution we can make along this line is to invite others to use the formal and empirical tools proposed here to challenge it. 


\section{ACKNOWLEDGEMENTS}

We thank Hervé Brailly, Louis Du Pasquier, Jonathan Ewbank, Michel Fougereau, Philippe Kourilsky, Leïla Périé, Bernard Malissen, and Alain Trautmann for their constructive comments, Sophie Guia and Sophie Ugolini for their experimental data on calcium flux, and Charles Chapple for his editorial help. E.V lab is supported by the European Research Council (THINK Advanced Grant) and by institutional grants from INSERM, CNRS and Aix Marseille to CIML. T.P and E.V. are scholars of the Institut Universitaire de France. E.V. is cofounder and shareholder of Innate Pharma. The other authors declare no conflict of interest. 


\section{Figures:}

\section{Figure 1:}

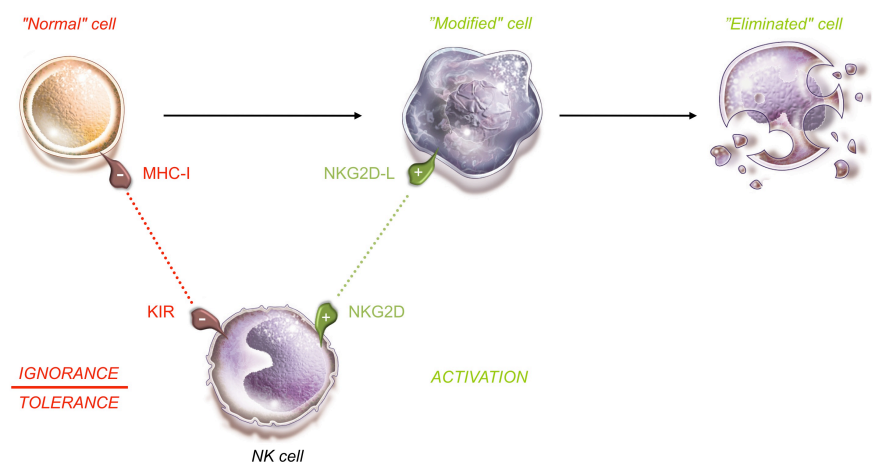

Fig.1A

Chronic presence of activating ligands

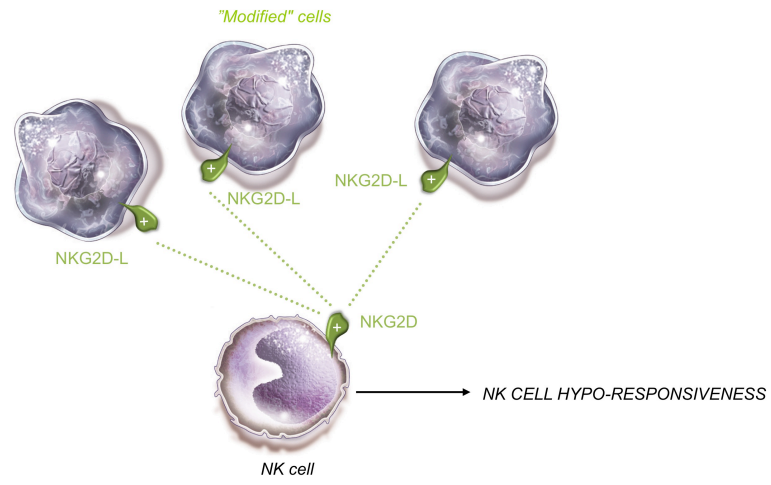

Fig.1B

Chronic absence of MHC-I recognition

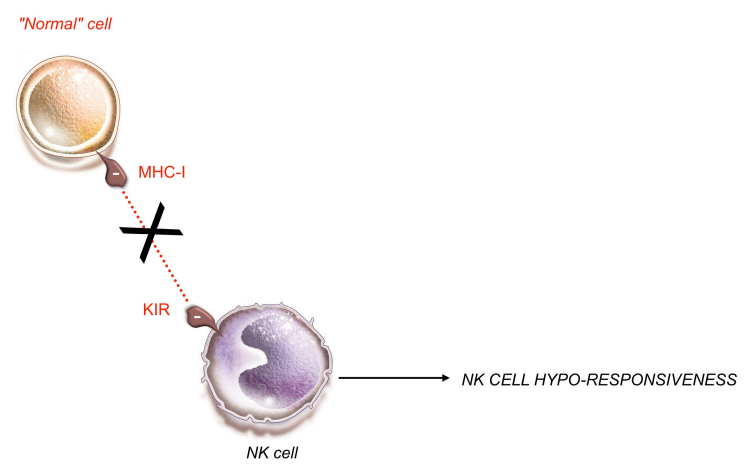

Fig.1C 


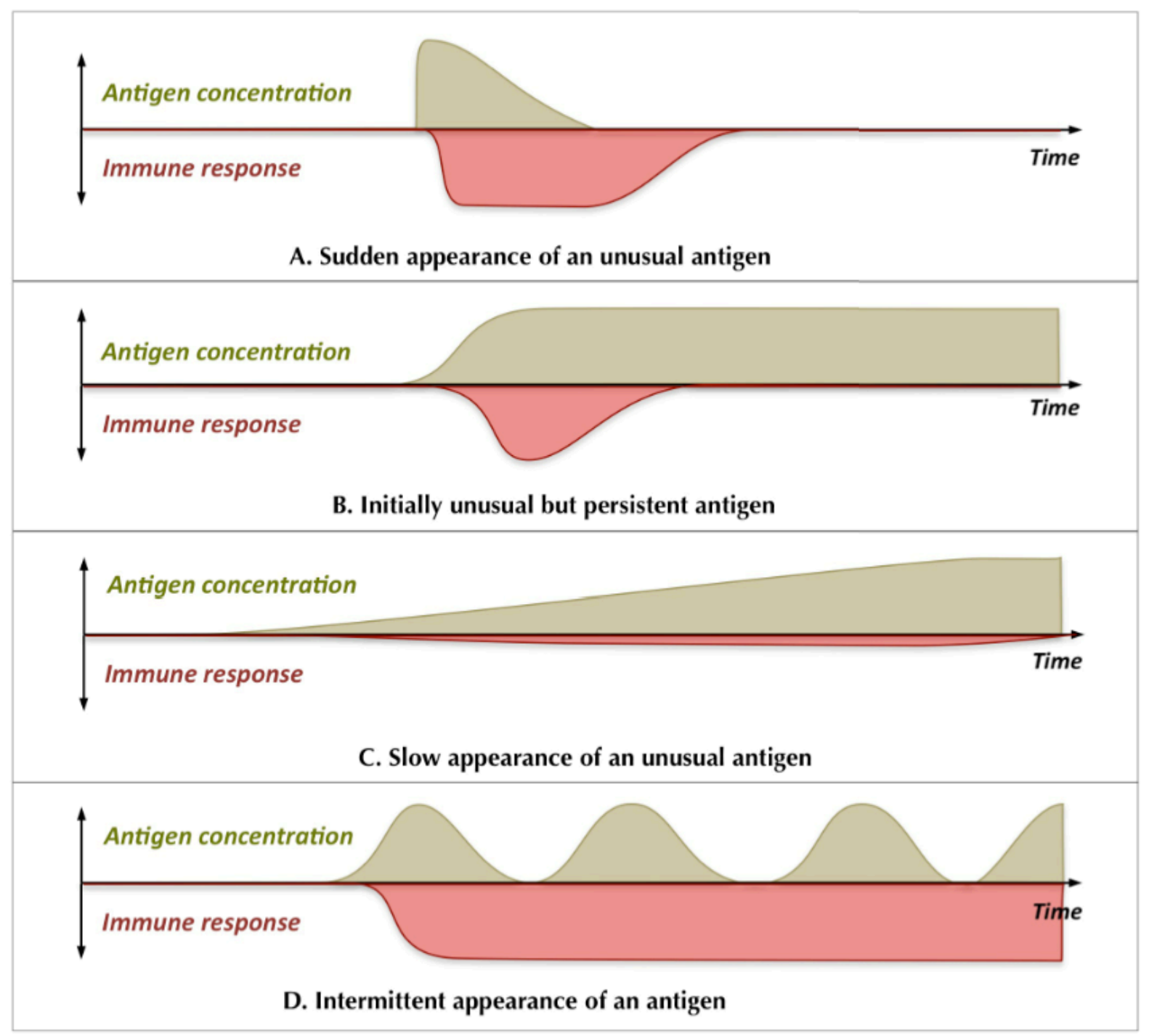

Pradeu et al., Figure 2

\section{Figure legends:}

\section{Figure 1. NK cell tuning}

Fig. 1A: NK cells can distinguish their targets from healthy cells through the engagement of activating receptors and the lack of engagement of inhibitory receptors. In this example, the recognition of MHC-I molecules on healthy cells by the inhibitory KIR expressed on human NK cells induces a dominant inhibitory signal in NK cells. In contrast, cells can be modified as the result of various assaults such as tumor transformation or microbial infection. In these circumstances, molecules induced or up-regulated at the surface of the target cell, engage 
activating receptors (such as NKG2D) leading to NK cell activation and eventually to the elimination of the target cells.

Fig. 1B: Whereas NK cells are activated by modified cells expressing ligands for activating receptors, the chronic presence of activating ligands re-program NK cells that become hyporesponsive.

Fig. 1C: Although the absence of MHC-I expression on target cells unleashes NK cells from the inhibition induced by receptors such as KIR, NK cells adapt to the long lasting absence of MHC-I recognition. When MCH-I or KIR are not present, or when MHC-I and KIR do not interact, NK cells adapt to this environment by increasing their threshold of reactivity, leading to NK cell hypo-responsiveness.

\section{Figure 2. The triggering of an immune response according to the discontinuity theory}

The discontinuity theory states that the key to the triggering of an immune response is antigenic difference in a time-dependent context. If structurally different motifs appear suddenly (i.e., a strong quantitative difference with regard to time), then a vigorous immune response occurs (A), possibly followed by the generation of memory cells. In the case of a motif that is initially unusual but persists through time, the effector immune response is rapidly extinguished (B). If immune receptors interact with motifs that change very progressively (i.e., weak quantitative variation with regard to time), then the immune response is weak, and the motifs become tolerated (C). Finally, if a structurally different motif appears in an oscillating way, then a very strong and long-lasting immune response occurs (D).

\section{Boxes:}

\section{Box 1: The importance of theories in biology}


Today, theoretical thinking is often criticized for its excessive abstraction and its lack of utility in the everyday life of scientists. Yet, it has long been established that having a theoretical framework is indispensable to conduct fruitful research, and that even scientific observations and experiments that seem the most 'objective' are always, at least to some extent, influenced by theoretical views ${ }^{53,64,65}$. A theory can be defined as an organized set of testable explanatory and predictive statements. Some biological theories take the form of hierarchical mechanisms ${ }^{1}$, but even then they make possible the formulation of explanations and predictions. A purely descriptive biology (for instance, at the molecular level) would not give rise to true explanations and predictions, and as such it would not contribute significantly to the advancement of science. The formulation of theories helps scientists to determine where to look at, to interpret what they observe, and to develop a specific worldview. It also makes possible fruitful comparisons between different scientific fields ${ }^{66}$. Finally, it confronts scientists with the necessity to make bold predictions, with the risk of being proven wrong, but also with the conviction that the refutation of wrong theories is useful. Therefore, because theories are crucial in the formulation of explanations and in the construction of new experiments, it seems highly desirable that all fields of biology, including molecular ones such as immunology, aim at constructing new and testable theories.

\section{Box 2: A mathematical model of the discontinuity theory}

As can be seen in the following figures, our model accurately reproduces the behavior qualitatively illustrated in Figure 2.

(A). Discontinuity refers to a perturbation of any environmental signal detected by the system in time space. Since we are interested in the variations of the inputs rather than in their values, $R(t)$ will essentially be a function of the derivatives of $E(t)$ rather than $E(t)$ itself. Thus,

$R t=f d E t d t$ 
We considered variables' values at discrete time steps rather than a continuum in time and we suggested manipulating the discrete equivalent of a derivative quantity, the finite differences defined by:

$\Delta E t=E t-E(t-h) h$

where $\mathrm{h}$ denotes the time step. As a convention, we will take a time step equal to 1 , thus in our case:

$\Delta E t=E t-E(t-1)$

In addition, since a central point of the discontinuity theory is the notion of adaptation, we took into account memory effects, so that $\mathrm{R}(\mathrm{t})$ is not only a function of $\Delta E(t)$ at the current time step t, but also depends on $\Delta E$ values at previous time steps :

$R t=f \Delta E t, \Delta E t-1, \Delta E t-2, \ldots, \Delta E t-\delta$

where $\delta$ denotes the memory parameter.

In this first version, we thus propose the following function for $\mathrm{R}(\mathrm{t})$ :

$R t=T 1 \delta i=0 i=\delta|\Delta E(t-i)|$

where $T(x)$ is a transfer function modeling the response capacity of the cell. Indeed, most if not all experimental data on immune responses triggered by increasing activation intensities indicate a threshold at lower intensities and a saturation phenomenon at higher intensities.

We thus suggest using a sigmoidal transfer function given by:

$T x=\alpha 1+e-\mu(x-\tau)$

where the coefficient $\alpha$ will set the amplitude order of the response $\mathrm{R}(\mathrm{t})$ while $\mu$ and $\tau$ will determine the saturation range and the threshold at lower values of $\mathrm{E}(\mathrm{t})$. They will eventually have to be set using experimental data and will depend on the cell type considered, but it is not of great importance at this qualitative level. $\mathrm{R}(\mathrm{t})$ presents all properties that we wanted to 
model. Indeed, $\mathrm{R}(\mathrm{t})$ will depend only on $\mathrm{E}(\mathrm{t})$ variations, will be high when these variations are sharp, and will return to zero as soon as $\mathrm{E}(\mathrm{t})$ is stable on the $\delta$ previous time steps which will correspond to the adaptation phenomenon.

The transfer function $\mathrm{T}(\mathrm{x})$ will model both activation threshold and saturation effects of the cell reactivity, and will set the amplitude of the response through the parameter $\alpha$. In this example, $\alpha=100, \mu=1$ and $\tau=5$.

(B). Comparison of the model with experimental data. Upper panel: $\mathrm{Ca}^{2+}$ flux changes in freshly isolated mouse splenic NK cells measured by flow cytometry as previously described (S. Guia et al. 2011). Briefly, biotinylated NKG2D mAb (CX5) was added to the cells followed by streptavidin to induce NKG2D cross-linking. The stimulation is presented as the ratio of Indo-1 (violet) to Indo-1 (blue). Lower panel: immune cell response profile $\mathrm{R}(\mathrm{t})$ predicted (on 3000 time steps) by the model for an entry function, which fits with the experimental data presented in the upper panel. This response profile was obtained with parameter values: and. In the lower panel and in panels $\mathrm{C}$ through $\mathrm{E}$, the numbers on the $\mathrm{y}$ axis are the values of $\mathrm{R}(\mathrm{t})$ (red line) and $\mathrm{E}(\mathrm{t})$ (grey line).

(C). Immune cell responses $R(t)$, as a function of a Poissonian input profile $[E t=\beta \lambda t e-\lambda t]$ that could be associated with typical dynamics of external perturbations like infections and any type of injection.

(D). Immune cell responses $\mathrm{R}(\mathrm{t})$, as a function of a sigmoid input profile $[\mathrm{E}(\mathrm{t})=\beta /(1+e-\lambda \mathrm{t})]$ that could be associated with typical dynamics of endogenous perturbations (interleukin production for instance).

(E). Immune cell responses $\mathrm{R}(\mathrm{t})$, as a function of an oscillating input profile $[E t=\beta(1+\cos \omega t)]$ modelling an intermittent antigen exposure. 

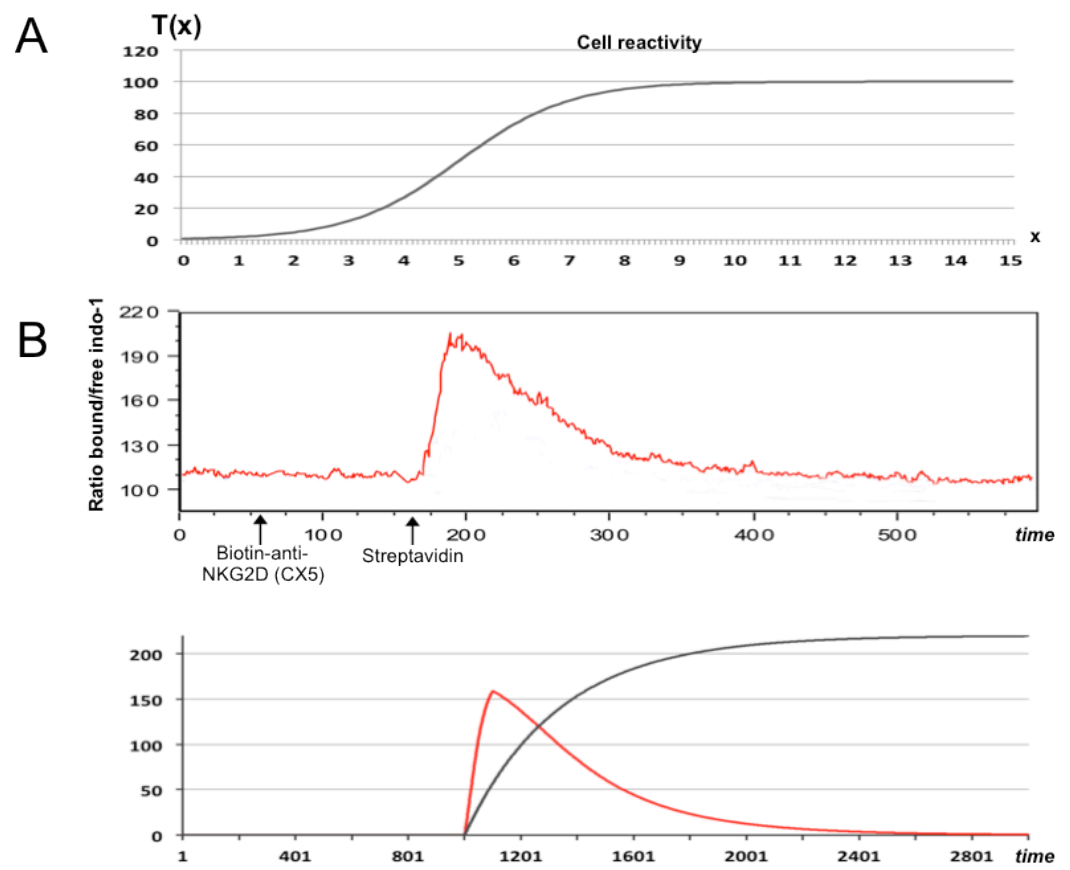

C



D

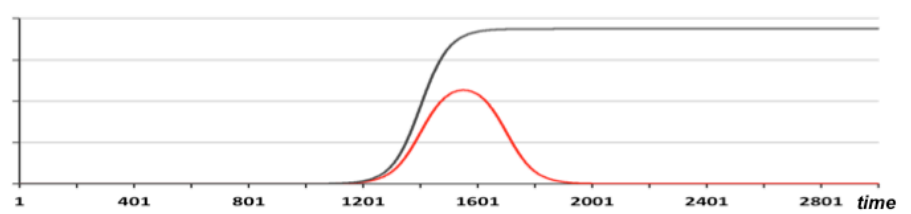

E

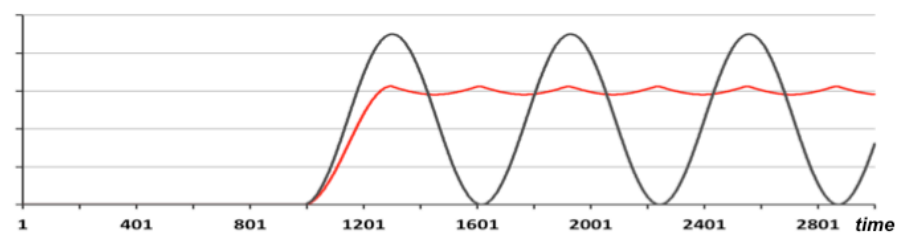

Pradeu et al., Figure 3 
${ }^{1}$ Craver, C. F. "Structures of Scientific Theories", in Blackwell Guide to the Philosophy of Science (ed.

Machamer, P. K. and Silberstein, M.), 55-79 (Blackwell, Oxford, 2001).

${ }^{2}$ Greenspan, N. S. Conceptualizing immune responsiveness. Nat. Immunol. 8, 5-7 (2007).

${ }^{3}$ Vivier, E. et al. Innate or Adaptive Immunity? The Example of Natural Killer Cells. Science 331, 44-49 (2011).

${ }^{4}$ Orr, M. T. \& Lanier, L. L. Natural killer cell education and tolerance. Cell 142, 847-856 (2010).

${ }^{5}$ Gasser, S. \& Raulet, D. H. Activation and self-tolerance of natural killer cells. Immunol. Rev. 214, 130-142 (2006).

${ }^{6}$ Kärre, K., Ljunggren, H. G., Piontek, G. \& Kiessling, R. Selective rejection of H-2-deficient lymphoma variants suggests alternative immune defence strategy. Nature 319, 675-678 (1986).

${ }^{7}$ Höglund, P. et al. Recognition of beta 2-microglobulin-negative (beta $2 \mathrm{~m}$-) T-cell blasts by natural killer cells from normal but not from beta $2 \mathrm{~m}$ - mice: nonresponsiveness controlled by beta $2 \mathrm{~m}$ - bone marrow in chimeric mice. Proc. Natl. Acad. Sci. U.S.A. 88, 10332-10336 (1991).

${ }^{8}$ Liao, N. S., Bix, M., Zijlstra, M., Jaenisch, R. \& Raulet, D. MHC class I deficiency: susceptibility to natural killer (NK) cells and impaired NK activity. Science 253, 199-202 (1991).

${ }^{9}$ Joncker, N. T., Shifrin, N., Delebecque, F. \& Raulet, D. H. Mature natural killer cells reset their responsiveness when exposed to an altered MHC environment. J Exp Med 207, 2065-2072 (2010).

${ }^{10}$ Elliott, J. M., Wahle, J. A. \& Yokoyama, W. M. MHC class I-deficient natural killer cells acquire a licensed phenotype after transfer into an MHC class I-sufficient environment. J Exp Med 207, 2073-2079 (2010).

${ }^{11}$ Savill, J., Dransfield, I., Gregory, C. \& Haslett, C. A blast from the past: clearance of apoptotic cells regulates immune responses. Nat Rev Immunol 2, 965-975 (2002).

${ }^{12}$ Stuart, L. M. \& Ezekowitz, R. A. Phagocytosis and comparative innate immunity: learning on the fly. Nat Rev Immunol 8, 131-141 (2008).

${ }^{13}$ Green, D. R., Ferguson, T., Zitvogel, L. \& Kroemer, G. Immunogenic and tolerogenic cell death. Nat Rev Immunol 9, 353-363 (2009).

${ }^{14}$ Jeannin, P., Jaillon, S. \& Delneste, Y. Pattern recognition receptors in the immune response against dying cells. Current Opinion in Immunology 20, 530-537 (2008).

${ }^{15}$ Ahrens, S. et al. F-Actin Is an Evolutionarily Conserved Damage-Associated Molecular Pattern Recognized by DNGR-1, a Receptor for Dead Cells. Immunity 36, 635-645 (2012).

${ }^{16}$ Foster, S. L., Hargreaves, D. C. \& Medzhitov, R. Gene-specific control of inflammation by TLR-induced chromatin modifications. Nature 447, 972-978 (2007).

${ }^{17}$ Wang, H. et al. Lack of CD47 on nonhematopoietic cells induces split macrophage tolerance to CD47null cells. Proceedings of the National Academy of Sciences 104, 13744-13749 (2007).

${ }^{18}$ Ramsdell, F. \& Fowlkes, B. J. Maintenance of in vivo tolerance by persistence of antigen. Science 257, 11301134 (1992).

${ }^{19}$ Goodnow, C. C. et al. Altered immunoglobulin expression and functional silencing of self-reactive B lymphocytes in transgenic mice. Nature 334, 676-682 (1988).

${ }^{20}$ Virgin, H. W., Wherry, E. J. \& Ahmed, R. Redefining chronic viral infection. Cell 138, 30-50 (2009).

${ }^{21}$ Utzschneider, D. T. et al. T cells maintain an exhausted phenotype after antigen withdrawal and population reexpansion. Nat Immunol 14, 603-610 (2013).

${ }^{22}$ Turchinovich, G. \& Hayday, A. C. Skint-1 Identifies a Common Molecular Mechanism for the Development of Interferon- $\gamma$-Secreting versus Interleukin-17-Secreting $\gamma \delta$ T Cells. Immunity 35, 59-68 (2011).

${ }^{23}$ Grossman, Z. \& Paul, W. E. Adaptive cellular interactions in the immune system: the tunable activation threshold and the significance of subthreshold responses. Proceedings of the National Academy of Sciences 89, 10365-10369 (1992).

${ }^{24}$ Grossman, Z., Min, B., Meier-Schellersheim, M. \& Paul, W. E. Concomitant regulation of T-cell activation and homeostasis. Nat Rev Immunol 4, 387-395 (2004).

${ }^{25}$ Schenten, D., and Medzhitov, R. The control of adaptive immune responses by the innate immune system. Advances in immunology 109, 87-124 (2011).

${ }^{26}$ Shankaran, V. et al. IFN $\gamma$ and lymphocytes prevent primary tumour development and shape tumour immunogenicity. Nature 410, 1107-1111 (2001).

${ }^{27}$ Galon, J. et al. Type, Density, and Location of Immune Cells Within Human Colorectal Tumors Predict Clinical Outcome. Science 313, 1960-1964 (2006). 
${ }^{28}$ Smyth, M. J., Dunn, G. P. \& Schreiber, R. D. in Advances in Immunology (James P. Allison, G. D.) Volume 90, 1-50 (Academic Press, 2006).

${ }^{29}$ Raulet, D. H. \& Guerra, N. Oncogenic stress sensed by the immune system: role of natural killer cell receptors, Nat Rev Immunol 9, 568-580 (2009).

${ }^{30}$ Kraman, M. et al. Suppression of Antitumor Immunity by Stromal Cells Expressing Fibroblast Activation Protein- $\alpha$. Science 330, 827-830 (2010).

${ }^{31}$ Pardoll, D. Does the immune system see tumors as foreign or self? Annu. Rev. Immunol. 21, 807-839 (2003).

${ }^{32}$ den Boer, A. T. et al. The Tumoricidal Activity of Memory CD8+ T Cells Is Hampered by Persistent Systemic Antigen, but Full Functional Capacity Is Regained in an Antigen-Free Environment. The Journal of Immunology 172, 6074-6079 (2004).

${ }^{33}$ Drake, C. G., Jaffee, E. \& Pardoll, D. M. Mechanisms of Immune Evasion by Tumors. Advances in Immunology Volume 90, 51-81 (2006).

${ }^{34}$ Pradeu, T. \& Carosella, E. D. On the definition of a criterion of immunogenicity. Proceedings of the National Academy of Sciences USA 103, 17858-17861 (2006).

${ }^{35}$ Burnet, F. M. Cancer: a biological approach. British Medical Journal 1, 1-7 (1957).

${ }^{36}$ Thomas, L. in Cellular and humoral aspects of the hypersensitive states (Lawrence, H. S.) 529-532 (HoeberHarper, 1959).

${ }^{37}$ Burnet, F. M. Immunological surveillance. (Pergamon, 1970).

${ }^{38}$ Zinkernagel, R. M. \& Doherty, P. C. Immunological surveillance against altered self components by sensitised T lymphocytes in lymphocytes choriomeningitis. Nature 251, 547-548 (1974).

${ }^{39}$ Houghton, A. N. Cancer antigens: immune recognition of self and altered self. The Journal of Experimental Medicine 180, 1-4 (1994).

${ }^{40}$ Laughlin, S. B. The role of sensory adaptation in the retina. Journal of Experimental Biology 146, 39-62 (1989).

${ }^{41}$ Wark, B., Lundstrom, B. N. \& Fairhall, A. Sensory adaptation. Current Opinion in Neurobiology 17, 423-429 (2007).

${ }^{42}$ Bray, D., Levin, M. D. \& Morton-Firth, C. J. Receptor clustering as a cellular mechanism to control sensitivity. Nature 393, 85-88 (1998).

${ }^{43}$ Henrickson, S. E. et al. T cell sensing of antigen dose governs interactive behavior with dendritic cells and sets a threshold for T cell activation. Nat Immunol 9, 282-291 (2008).

${ }^{44}$ Apostolou, I. \& von Boehmer, H. In Vivo Instruction of Suppressor Commitment in Naive T Cells. The Journal of Experimental Medicine 199, 1401-1408 (2004).

${ }^{45}$ Kyes, S., Horrocks, P. \& Newbold, C. Antigenic variation at the infected red cell surface in malaria. Annu. Rev. Microbiol. 55, 673-707 (2001).

${ }^{46}$ MacGregor, P., Szöőr, B., Savill, N. J. \& Matthews, K. R. Trypanosomal immune evasion, chronicity and transmission: an elegant balancing act. Nat Rev Micro 10, 431-438 (2012).

${ }^{47}$ Ndung'u, T. \& Weiss, R. A. On HIV diversity. AIDS 26, 1255-1260 (2012).

${ }^{48}$ Csikász-Nagy, A. \& Soyer, O. S. Adaptive dynamics with a single two-state protein. Journal of The Royal Society Interface 5, S41-S47 (2008).

${ }^{49}$ Daëron, M., Jaeger, S., Du Pasquier, L. \& Vivier, E. Immunoreceptor tyrosine-based inhibition motifs: a quest in the past and future. Immunol. Rev. 224, 11-43 (2008).

${ }^{50}$ Hori, S., Nomura, T. \& Sakaguchi, S. Control of regulatory T cell development by the transcription factor Foxp3. Science 299, 1057-1061 (2003).

51 Fontenot, J. D., Gavin, M. A. \& Rudensky, A. Y. Foxp3 programs the development and function of CD4+CD25+ regulatory T cells. Nat. Immunol. 4, 330-336 (2003).

${ }^{52}$ Khattri, R., Cox, T., Yasayko, S.-A. \& Ramsdell, F. An essential role for Scurfin in CD4+CD25+ T regulatory cells. Nat. Immunol. 4, 337-342 (2003).

${ }^{53}$ T. S. Kuhn, The Structure of Scientific Revolutions (University of Chicago Press, Chicago, 1962).

${ }^{54}$ Matzinger, P. The danger model: A renewed sense of self. Science 296, 301-305 (2002).

${ }^{55}$ Hooper, L. V., Littman, D. R. \& Macpherson, A. J. Interactions Between the Microbiota and the Immune System. Science 336, 1268-1273 (2012).

${ }^{56}$ Lathrop, S. K. et al. Peripheral education of the immune system by colonic commensal microbiota. Nature 478, 250-254 (2011).

${ }^{57}$ Belkaid, Y. \& Oldenhove, G. Tuning Microenvironments: Induction of Regulatory T Cells by Dendritic Cells. Immunity 29, 362-371 (2008).

${ }^{58}$ Shklovskaya, E. et al. Langerhans cells are precommitted to immune tolerance induction. Proceedings of the National Academy of Sciences 108, 18049-18054 (2011).

${ }^{59}$ Carrat, F. \& Flahault, A. Influenza vaccine: The challenge of antigenic drift. Vaccine 25, 6852-6862 (2007). 
${ }^{60}$ Pradeu, T. The Limits of the Self: Immunology and Biological Identity. (Oxford University Press, New York, 2012).

${ }^{61} \mathrm{Du}$ Pasquier, L. \& Bernard, C. C. A. Active Suppression of the Allogeneic Histocompatibility Reactions During the Metamorphosis of the Clawed Toad Xenopus. Differentiation 16, 1-7 (1980).

${ }^{62}$ Hailemichael, Y. et al. Persistent antigen at vaccination sites induces tumor-specific CD8+ T cell sequestration, dysfunction and deletion. Nat Med 19, 465-472 (2013).

${ }^{63}$ Burks, A. W. et al. Oral Immunotherapy for Treatment of Egg Allergy in Children. N Engl J Med 367, 233243 (2012).

${ }^{64}$ Bernard, C. Experimental Medicine. (Transaction, New Brunswick, 1999) (original text published in 1865).

${ }^{65}$ Popper, K. R. Logik Der Forschung: Zur Erkenntnistheorie Der Modernen Naturwissenschaft. (J. Springer, Wien, 1935).

${ }^{66}$ N. R. C., The Role of Theory in Advancing 21st-Century Biology: Catalyzing Transformative Research (The National Academies Press, 2008; http://www.nap.edu/openbook.php?record id=12026). 